\title{
Espiritualidad y estilo de vida en estudiantes de Medicina Humana de la Universidad Peruana Unión, Lima 2017
}

\author{
Spirituality and lifestyle in Human Medicine students of Universidad Peruana Unión, \\ Lima 2017
}

\section{Lea Rosmery Alva Rosnay ${ }^{1}$, Luz Castillo Zamora²}

\begin{abstract}
RESUMEN
Objetivo: Determinar la relación entre la espiritualidad y estilo de vida en los estudiantes de Medicina Humana. Materiales y Método: De enfoque cuantitativo, diseño no experimental, tipo correlacional y de corte transversal. La selección de la muestra se realizó mediante el muestreo no probabilístico por conveniencia y estuvo conformada por 176 estudiantes. El cuestionario empleado para la recolección de datos fue el "cuestionario de espiritualidad" creado por Parsian y Dunning (2009) y traducido y adaptado al español por Díaz (2012). A su vez, para medir la variable estilos de vida, se utilizó el cuestionario "S.N. - 52" creado por Walker, Sechrist y Pender (1996). Cabe resaltar que ambos instrumentos presentaron un coeficiente de Alpha de Cronbach alto con un valor de 0,9 , demostrando su confiabilidad. Resultados: Se encontró relación entre la espiritualidad y el estilo de vida con un p-valor de 0, 002, según la prueba estadística Chi-cuadrado. Del mismo modo, se encontró relación entre las dimensiones de la espiritualidad y el estilo de vida con un p-valor $<0,05$. Conclusión: Existe relación entre las variables de estudio.
\end{abstract}

Palabras clave: Espiritualidad, estilo de vida, identidad interior, sentido de vida.

\begin{abstract}
Objective: To determine the relationship between spirituality and lifestyle in students of Human Medicine. Materials and methods: Quantitative approach, non-experimental design, correlation type and cross-sectional type. The selection of the sample was made by non-probabilistic sampling for convenience and was made up of 176 students. The questionnaire used for data collection was the "spirituality questionnaire" created by Parsian and Dunning (2009) and translated and adapted into Spanish by Diaz (2012). In turn, to measure the lifestyle variable, it was used the "SN - 52" questionnaire, created by Walker, Sechrist, and Pender (1996). It should be noted that both instruments presented a high Cronbach Alpha coefficient with a value of 0.9 , demonstrating their reliability. Results: There was a relationship between spirituality and the lifestyle with a p-value of 0.002 , according to the Chi-square statistical test. Similarly, a relationship was found between the dimensions of spirituality and lifestyle with a $p$-value $<0.05$. Conclusion: There is a relationship between the study variables.
\end{abstract}

Keywords: Spirituality, lifestyle, inner identity, the meaning of life. 


\section{INTRODUCCIÓN}

La Organizacion Mundial de la Salud (2016) refiere que: "la salud es un estado de completo bienestar físico, mental y social, y no solamente la ausencia de afecciones o enfermedades". Sin embargo, en estos últimos años, el avance de la tecnología, el exceso de trabajo y de los estudios, han modificado los estilos de vida a tal punto que llegan a ser perjudiciales para la persona, no permitiéndoles alcanzar un estado de salud total.

Con respecto a esto, Bastias y Stiepovich (2014) afirman que "los avances científicos han propiciado que la sociedad experimente cambios importantes en los indicadores vinculados a la salud. Si bien, estos han permitido un incremento en la esperanza de vida al nacer, de la población mundial, también han significado la aparición de problemas de salud emergentes que pudiesen ser más difíciles de erradicar que los que afectaban antaño, pues su raíz radica en los estilos de vida de las personas".

A nivel mundial, la población juvenil está creciendo en términos tanto absolutos como relativos. Más de la mitad de la población presenta menos de 25 años de edad y aquellos entre 10 y 24 años constituyen alrededor de 1.600 millones (28\%), de los cuales el $85 \%$ habita en países del tercer mundo (Sistema de las Naciones Unidas en el Perú, 2013). Asimismo, esta población joven ha venido presentando cambios negativos en la conducta, tales como el sedentarismo y la mala alimentación, generando permutas en el estilo de vida y el aumento de enfermedades no transmisibles. A su vez, la vida moderna trajo consigo el desequilibrio de varios factores determinantes para la salud y la calidad de vida del individuo, la descomposición del medio familiar y social, inestabilidad afectiva y emocional, deterioro del ambiente urbano, agresividad y violencia dados por el avance de la globalización, el aumento de la propaganda y la intensa campaña de preconsumo exponiendo a la población a cambios en las condiciones socioeconómicas y en los estilos de vida (Castro y Cabrera - CEDRO; 2007).

Por tanto, intervenir en el estilo de vida se convierte en una de las acciones más eficaces para la prevención de la enfermedad y la promoción de la salud. Según Ponte (2010) este tema debe ser abordado desde espacios universitarios para avanzar en la educación integral de los futuros profesionales, más aún en estudiantes de las ciencias médicas, ya que conocen de la relación entre el comportamiento y la salud, así como el origen de las enfermedades y su tratamiento que les permitirán evitar malas prácticas saludables.

De igual manera, De la Calle (2010) señala que "es también misión de la universidad motivar al estudiante, desde una actitud de sincero respeto a su libertad, para que profundice en sus creencias religiosas y busque desarrollar este ámbito fundamental de su persona". No obstante, Florenzano (2010) indica que "aún existe cierto recelo en el estudio de esta área, ya que se confunde religión con puntos de vista intolerantes, dogmáticos u oscurantistas". A pesar de ello, la concientización sobre el bienestar espiritual como una dimensión más del estado de salud, que favorece los estilos de vida y comportamientos más sanos, lo que se asociaría a un menor riesgo de enfermedades y a una actitud diferente cuando se pierde la salud (Salgado, 2015)

Por lo tanto, el estudio tiene como hipótesis: Existe relación entre espiritualidad y estilo de vida en estudiantes de Medicina Humana de la Universidad Peruana Unión.

\section{MATERIALES Y MÉTODO}

Se realizó una investigación de enfoque cuantitativo, diseño no experimental, tipo correlacional y de corte transversal.

La población estuvo conformada por 372 estudiantes, del primer y segundo año de la carrera de medicina humana. Para la selección de la muestra, se utilizó el muestreo no probabilístico, por conveniencia y considerando criterios de inclusión y exclusión, quedando un total de 176 participantes.

Para medir la variable estilos de vida, se empleó el cuestionario estandarizado "S.N. - 52" creado por Walker, Sechrist y Pender (1995) que contó con una confiabilidad de 0,9 , a través de coeficiente Alpha de Cronbach. Asimismo, se utilizó el "Cuestionario de Espiritualidad" elaborado por Parsian y Dunning (2009) que también contó con una confiabilidad de 0,9. 


\section{RESULTADOS}

Tabla 1.

Datos sociodemográficos

Características sociodemográficas de los estudiantes de Medicina Humana de la Universidad Peruana Unión.

\begin{tabular}{|c|c|c|c|}
\hline Variable & Escala & $\mathbf{n}$ & $\%$ \\
\hline \multirow[t]{3}{*}{ Edad } & Adolescente segunda fase $(15-19)$ & 100 & 56,8 \\
\hline & Adulto en transición (20 -24) & 69 & 39,2 \\
\hline & Adulto intermedio (25 - 54) & 7 & 4,0 \\
\hline \multirow[t]{2}{*}{ Sexo } & Femenino & 100 & 56,8 \\
\hline & Masculino & 76 & 43,2 \\
\hline \multirow[t]{5}{*}{ Ciclo de estudio } & 2 & 18 & 10,2 \\
\hline & 3 & 80 & 45,5 \\
\hline & 4 & 27 & 15,3 \\
\hline & 5 & 49 & 27,8 \\
\hline & 6 & 2 & 1,1 \\
\hline \multirow[t]{4}{*}{ Procedencia } & Costa & 93 & 52,8 \\
\hline & Sierra & 34 & 19,3 \\
\hline & Selva & 8 & 4,5 \\
\hline & Extranjero & 41 & 23,3 \\
\hline \multirow[t]{4}{*}{ Vive con } & Padres & 63 & 35,8 \\
\hline & Familiares & 5 & 2,8 \\
\hline & Pensión Externa & 73 & 41,5 \\
\hline & Internado Universitario & 35 & 19,9 \\
\hline \multirow[t]{3}{*}{ Religión } & Adventista & 154 & 87,5 \\
\hline & Católico & 3 & 1,7 \\
\hline & Otro & 19 & 10,8 \\
\hline \multirow[t]{2}{*}{ Bautizado } & Sí & 153 & 86,9 \\
\hline & No & 23 & 13,1 \\
\hline \multirow{4}{*}{$\begin{array}{l}\text { Asistencia a la } \\
\text { iglesia }\end{array}$} & Siempre & 125 & 71,0 \\
\hline & Frecuentemente & 29 & 16,5 \\
\hline & A veces & 21 & 11,9 \\
\hline & Nunca & 1 & 6 \\
\hline Total & & 176 & $100 \%$ \\
\hline
\end{tabular}

Tabla 2

Nivel de Espiritualidad en los estudiantes de Medicina Humana de la Universidad Peruana Unión, 2017.

\begin{tabular}{|cll} 
Nivel de Espiritualidad & $\mathbf{n}$ & $\%$ \\
\hline Alto & 44 & 25,0 \\
Bajo & 132 & 75,0 \\
Total & 176 & 100,0 \\
\hline
\end{tabular}


Tabla 3

Nivel de Estilo de Vida en los estudiantes de Medicina Humana de la Universidad Peruana Unión, 2017.

\begin{tabular}{lll} 
Nivel de Estilo de Vida & $\mathrm{n}$ & $\%$ \\
No saludable & 141 & 80,1 \\
Saludable & 35 & 19,9 \\
Total & 176 & 100,0 \\
\hline
\end{tabular}

Tabla 4

Relación entre el Nivel de Espiritualidad y el Estilo de Vida en los estudiantes de Medicina Humana de la Universidad Peruana Unión, 2017.

\begin{tabular}{llllll} 
& & & \multicolumn{3}{c}{ Estilo de Vida } \\
\cline { 3 - 6 } & & & No saludable & Saludable & Total \\
\cline { 5 - 6 } & Alto & $\mathrm{n}$ & 28 & 16 & 44 \\
Nivel de & $\%$ & $15.9 \%$ & $9.1 \%$ & $25,0 \%$ \\
Espiritualidad & Bajo & $\mathrm{n}$ & 113 & 19 & 132 \\
& & $\%$ & $64,2 \%$ & $10,8 \%$ & $75,0 \%$ \\
& Total & $\mathrm{n}$ & 141 & 35 & 176 \\
& & $\%$ & $80,1 \%$ & $19,9 \%$ & $100,0 \%$ \\
\hline
\end{tabular}

${ }^{*} x^{2}:$ 9,998a, $p$ - valor: ,002

Tabla 5

Relación entre las dimensiones y Estilo de Vida en los estudiantes de Medicina Humana de la Universidad Peruana Unión, 2017.

\begin{tabular}{lll} 
Dimensiones & Chi cuadrado & P \\
Identidad interior & $10,068^{\mathrm{a}}$ & 0,002 \\
Sentido de vida & $13,463^{\mathrm{a}}$ &, 000 \\
Conexión con las personas & $5,137^{\mathrm{a}}$ & 0,023 \\
\hline
\end{tabular}

\section{DISCUSIÓN}

El avance de la globalización, y del mundo contemporáneo, deja notar que los jóvenes universitarios están cada vez más expuestos a múltiples factores de riesgo psicosocial, de los cuales no están muy conscientes (Castro y Llanes, 2006).

Los datos descriptivos del estudio revelaron que el $75 \%$ de los estudiantes de medicina manifestaron un nivel de espiritualidad bajo y solo el $25 \%$, alto. A su vez, el $80 \%$ de los mismos, indicaron un estilo de vida no saludable, y el $19,9 \%$ saludable. Resultados similares reportaron Tacilla y Robles (2015) en su trabajo "Felicidad y espiritualidad en miembros de una iglesia cristiana de Lima-Este", donde el $98.1 \%$ de los participantes jóvenes indicaron una espiritualidad elevada y solo la minoría baja.
Por otro lado, Piedmont (2007) indica que "la parte espiritual es de suma importancia en cuanto al análisis y comprensión de la calidad de vida, aportando significado a sus vidas y posibilitando a las personas a que se conecten con un propósito mayor". Es así que las personas que presentan altos valores de trascendencia espiritual, teniendo una visión de la vida más holística que les permite percibir la unidad con todos los elementos de la naturaleza, así como también sincronicidad en sus vidas, por lo que asumen un verdadero compromiso con los demás.

Al analizar la relación entre ambas variables, se encontró que existe relación entre la espiritualidad y el estilo de vida con un p-valor $<0,05$. Resultados similares reportaron Nelms, Hutchins, Hutchins y Pursley (2007) en su investigación: "La espiritualidad y la salud de los 
estudiantes universitarios", donde hallaron una correlación positiva de rho de Spearman $(r=$ $.180 ; p=.026)$.

Seguidamente, Kennedy (2002) resalta que la espiritualidad está asociado positivamente al aumento significativo en la percepción del bienestar en la vida, la autoconfianza en el manejo de problemas y una tendencia en la disminución del enfado o enojo. De igual manera, Quiceno y Vinaccia (2009) argumentan que un gran grupo de familiares y pacientes incluyen la parte espiritual como punto de vital importancia en la salud y en sus vidas, así también que la espiritualidad tiene una influencia a nivel físico en la persona, ya es capaz de producir o influenciar en los cambios fisiológicos que mejoren la salud de la persona.

Según D`Angelo (2002) la importancia del análisis del sujeto, para la construcción del sentido de vida, nos lleva a la cuestión de la autonomía y la praxis social que supondría un factor indispensable en el autocuidado de la salud y la práctica de hábitos de vida saludables.

Por otro lado, la personalidad saludable asume una conducta y actitud comunicativa, firme y abierta hacia la realidad en la que se vive y toma conciencia de que es un proceso continuo de aprendizaje, y se da mediante una interacción dialéctica con esta, el aprendizaje consiste en un cambio y trasformación para mejorar a mi mismo y a mi entorno (Gárciga y Alburquerque, 2012).

La conexión emocional puede permitir que la persona, al compartir experiencias, pueda mejorar o cambiar cosas positivas o negativas en su vida, cambios que puedan ayudarlo a llegar a tener una calidad de vida óptima.

Otro dato encontrado, en el estudio, demostró que sí existe relación entre las dimensiones de la espiritualidad con el estilo de vida; se halló relación entre la dimensión "sentido de vida" y el estilo de vida con un $\mathrm{p}$ - valor $<0,05$, asimismo, al analizar la relación entre la dimensión "identidad interior" y el estilo de vida se evidenció que sí existe relación con un $\mathrm{p}$ - valor $<0,05 \mathrm{y}$, por último, se encontró que existe relación entre la dimensión "conexión con las personas" y el estilo de vida, con un $p-$ valor $<0,05$.

Se puede decir que las relaciones significativas son la base fundamental para la relación del individuo con los demás y con la sociedad, la base de la comunidad, el sentido y la finalidad procedentes de temas de la vida, un sentido de relación y la promoción de los vínculos interpersonales. La identidad de la persona tiene una relación estrecha con el estilo de vida que lleve, es por eso la importancia de poder contribuir en esta dimensión a través de la espiritualidad, para así poder lograr formar mejores profesionales a futuro (Navas y Villegas, 2006).

Los estilos de vida que los adolescentes toman en esta etapa de transición, para su formación futura, siempre requiere de una guía de personas que integren su entorno, estas deben ayudar a tener conciencia sobre las consecuencias positivas y negativas que tendrán, de acuerdo a las decisiones tomadas. Todo lo que decidan se verá influenciado por la formación moral y ética que presenten, además de la influencia del entorno social; ya que, sin un buen guía, con dificultad lograrán alcanzar una vida adulta plena y saludable, porque no tendrán patrones de conductas que sean de ejemplo para otros. (Berra y Dueñas, 2008).

Finalmente, desde la perspectiva de la salud pública, y en particular de la conceptualización de los determinantes sociales en los procesos de salud-enfermedad, es importante considerar todo lo relacionado a la espiritualidad y sopesar esta como un punto más dentro de la educación integral de los estudiantes. Del mismo modo, debemos tener en cuenta que esta dimensión puede llegar a intervenir en la parte promocional de la salud, promoviendo estilos de vida saludables en los estudiantes (Gómez y Cogollo, 2015).

\section{Declaración de financiamiento y de conflictos de interés:}

El estudio fue financiado por los autores, quienes declaran no tener conflictos de interés.

\section{Correspondencia}

Lea Rosmery Alva Rosnay.

Dirección: Clínica Ana Sthal, Iquitos, Perú

Correo electrónico: rosmeryrosnay@gmail.com 


\section{REFERENCIAS}

Bastias E. y Stiepovich J. (2014). Una revisión de los estilos de vida de estudiantes universitarios iberoamericanos. Ciencia y Enfermeria, 20(2), 93-101. Retrieved from http://www.scielo.cl/pdf/ cienf/v20n2/art_10.pdf

Castro. M y Llanes. J. (2006). Habilidades para la vida en estudiantes universitarios. LiberAddictus, 91, 1-9. Retrieved from www.infoadicciones.net

D`Angelo. O. (2002). Sentido de vida, sociedad y proyectos de vida. Ética y Sociedad, 2, 1-42. Retrieved from http://biblioteca.clacso.edu. ar/ar/libros/cuba/cips/caudales05/Caudales/ ARTICULOS/ArticulosPDF/07D054.pdf

De la Calle, C. (2010). La formación de la responsabilidad social del universitario: un estudio empírico. Universidad Complutense de Madrid, Madrid. Retrieved from http://eprints. ucm.es/10187/1/T31406.pdf

Florenzano, R. (2010). Religiosidad y salud mental: ¿amigos o enemigos? Revista de Gaceta de Psiquiatría Universitaria, 6(2), 221-229. Retrieved from http://revistagpu.cl/GPU 2 (2010)/ REF Religiosidad.pdf

Gárciga. O y Alburquerque . F. (2012). Las conductas adictivas: Análisis crítico y porpuestas para su prevención. Revista del Hospital Psiquiátrico de La Habana, 9(2). Retrieved from http://www. medigraphic.com/pdfs/revhospsihab/hph-2012/ hph122o.pdf

Gómez, E y Cogollo, Z. (2015). Asociación entre religiosidad y estilo de vida en adolescentes. Revista de la Facultad de Medicina, 63(2), 193-198. http://doi.org/10.15446/revfacmed. v63n2.49289

Kennedy.J, A. . y R. B. (2002). Changes in sprituality and well being in a retreat program for cardiac patients. Alternative Therapies In Health And Medicine, 8(4), 64-65. Retrieved from https:// jeksite.org/research/alther02.pdf

Navas. C y Villegas.K. (2006). Espiritualidad y salud. Revista Ciencias de la Educación, 1, 29-45. Retrieved from http://servicio.bc.uc.edu.ve/ educacion/revista/volln27/27-2.pdf

Nelms, H. y P. (2007). Spirituality and the health of college students. Journal of Religion and Health, 46(2), 249-265. http://doi.org/10.1007/s10943006-9075-0

Organización Mundial de la Salud. (2016). OMS | Salud de los adolescentes. Retrieved January 10,
2018, from http://www.who.int/topics/adolescent_ health/es/

Piedmont. (2007). Cross-cultural generalizability of the spiritual transcendence scale to the philippines: spirituality as a human universal. Mental Health, Religion and Culture, 10(2), 89-107. http://doi. org/10.1080/13694670500275494

Ponte M. (2010). Estilo de vida de los estudiantes de enfermería, segun año de estudio de la Universidad Nacional Mayor de San Marcos 2010. Universidad Peruana Unión. Retrieved from http://cybertesis.unmsm.edu.pe/bitstream/ cybertesis/1307/1/ponte_am.pdf

Quiceno M. y Vinaccia S. (2009). La salud en el marco de la psicología de la religión y la espiritualidad. Diversitas: Perspectivas en Psicología, 5(2), 321-336. Retrieved from http://www.redalyc.org/ pdf/679/67916260008.pdf

Salgado.A. (2015). Formación Universitaria en psicología de la religión y la espiritualidad: ¿Necesidad o Utopía? University training in psychology of religion and spirituality: a need or utopia? Revista Digital de Investigación En Docencia Universitaria, 2, 89-103. Retrieved from http://repositorio.usil.edu.pe/ bitstream/123456789/1781/1/2015_Salgado_ Formacion universitaria en psicologia de la religion y espiritualidad.pdf

Sistema de las Naciones Unidas en el Peru. (2013). Sistema de las Naciones Unidas en el Perú. Retrieved February 2, 2018, from http://onu.org. pel

Tacilla S. y Robles M. (2015). Felicidad y espiritualidad en miembros de una iglesia cristiana de Lima Este, 2015. Universidad Peruana Unión. Retrieved from http://repositorio.upeu.edu.pe/ bitstream/handle/UPEU/175/Mariella_Tesis bachiller_2015.pdf?sequence=1\&isAllowed=y

Recibido: 11/01/18

Aceptado: 23/04/18 